\title{
La gestión de la calidad en la Administración General del Estado
}

\section{Amador Elena Córdoba *}

En la antesala del siglo XXI es un hecho universalmente reconocido que las Administraciones Públicas constituyen un factor determinante en el desenvolvimiento social, económico, cultural, científico y técnico de cualquier país. El papel del Estado, a través de sus aparatos administrativos, es decisivo para el progreso y bienestar de las sociedades nacionales. Entre las variadas funciones de la Administración Pública destaca la de proveer a los ciudadanos bienes y servicios que sólo el Estado se encuentra en condiciones de garantizar en términos de equidad para el conjunto de la sociedad. Por su parte, los ciudadanos demandan cada vez más servicios y, al propio tiempo, exigen que sus aportaciones, ya sean directas o tributarias, se utilicen eficientemente. Los ciudadanos tienen, por tanto, derecho a recibir las prestaciones y servicios públicos con niveles de calidad adecuados a sus expectativas, derivadas de su doble condición de usuarios y contribuyentes.

\section{La Nueva Gestión Pública}

En la última década, tanto los gobiernos y administraciones de los distintos países como los estudiosos y expertos en la materia han dedicado esfuerzos a la búsqueda de nuevos modelos de gestión adaptados a las exigencias del entorno actual, en un proceso que se ha venido denominando de modernización. El conjunto de ideas, líneas y principios más o menos consolidados configura precisamente el paradigma de la Nueva Gestión Pública, como marco de investigación e iniciativas para la renovación de las organizaciones y la mejora de los servicios públiCOS (LÖFFLER, 1996).
A grandes rasgos y sin ánimo exhaustivo, los elementos que dibujan el contexto de la Nueva Gestión Pública son los siguientes:

* Entorno económico caracterizado por la globalización, la convergencia europea, la moderación del gasto público y, eventualmente, el proceso de reducción del tamaño del sector público.

* Entorno social caracterizado por la crisis de legitimidad de "lo público", la mayor presión ciudadana, manifestada como reequilibrio de posiciones ciudadano-Administración, y la demanda social de mantenimiento de una amplia presencia del sector público.

* Entorno político-institucional caracterizado por la multiplicidad de agentes e interlocutores, la necesidad de articular los ámbitos político y administrativo y, por lo que se refiere a la Administración General del Estado, redefinición de su Misión, para asumir, frente a las otras administraciones, una función eminentemente mediadora y redistribuidora hacia el conjunto de la sociedad nacional.

* Entorno cultural caracterizado, de una parte, por la aspiración de los gestores públicos a los valores de autonomía, responsabilidad y evaluación de resultados y, de otra, por el marco de referencia de la Calidad.

Conviene detenerse en este último aspecto, ya que hoy día Calidad es un concepto, diríase, omnímodo, un principio rector que impregna todas las facetas de todo tipo de organizaciones, ya sean éstas privadas o públicas, mercantiles o no lucrativas, industriales o de servicios. En última instancia, el factor determinante de esta situación reside en la fuerza y protagonismo adquiridos por los agentes - antes pasivos- del proceso económico: los consumidores, usuarios o clientes. Con todas las matizaciones que se deseen, en sus manos está el éxito o el fracaso, 
la supervivencia de una empresa. La Administración Pública, sistema de organizaciones eminentemente prestadoras de servicios, gana su legitimación contemporánea sobre todo respondiendo a los requerimientos que, como para el sector privado, emanan de los ciudadanos-usuarios. La Administración Pública no puede sustraerse a la implantación en su seno de la cultura de la calidad y sus técnicas de gestión, tanto en las relaciones directas con los usuarios como en el desarrollo de sus procesos internos en función de los mismos.

Sin entrar a analizar detenidamente la evolución del concepto de Calidad, interesa destacar que el predominio conquistado por el sector servicios en las economías avanzadas le ha imprimido el impulso y el sesgo definitivos como satisfacción del cliente. Las características propias del servicio frente al producto manufacturado han redefinido, en gran medida, el concepto de calidad e incorporado a la gestión de calidad las especificidades de la calidad de servicio.

Si se admite que la Administración Pública es por excelencia una organización prestadora de servicios, habrá de reconocerse la importancia vital e inaplazable de la adopción de un paradigma de gestión tan afianzado que, como postulaba un reciente artículo periodístico, la calidad ya no es una ventaja competitiva. Evidentemente, el marco de competencia, el mercado obliga a las empresas a mejorar continuamente. Pero la ausencia o relatividad de tal contexto para la Administración Pública no desvirtúa la pertinencia de su empeño por la mejora de la calidad de los servicios públicos, porque dispensar prestaciones y servicios adecuados a las expectativas de los ciudadanos que los financian con sus aportaciones deviene una exigencia para el Estado social y democrático de Derecho.

\section{Los principios del Servicio Público y la gestión de calidad}

Parece evidente que la calidad de la gestión pública depende de adoptar una gestión pública de la calidad. Ahora bien, si, como se señalaba más arriba, es en el escenario de modernización de la Administración Pública en el que se produce el rencuentro" con la Calidad como referente para la implantación de la Nueva Gestión Pública, resulta oportuno preguntarse por qué. No puede explicarse por una coincidencia accidental, ni por mimetismo gerencialista. Las líneas para determinar la pertinencia de la Gestión de Calidad deben partir de los principios y valores, de la misión y la visión de la Administración Pública actual. Se trataría de analizar si los principios, técnicas y herra- mientas de la Gestión de Calidad sirven para implementar los propios del Servicio Público.

De todos es sabido que el Servicio Público se encuentra guiado por una serie de principios, establecidos en el ordenamiento jurídico o comúnmente aceptados por la doctrina y la literatura especializada. Legalidad, igualdad, objetividad, continuidad, transparencia, participación, responsabilidad y eficacialeficiencia. Estos principios sobradamente conocidos, como señala la exposición de motivos de la Ley 6/1997, de 14 de abril, de Organización y Funcionamiento de la Administración General del Estado, adquieren plena virtualidad por su interrelación sinérgica y complementados por otro de carácter finalista. El destinatario del servicio público, de la actividad administrativa no es otro que el ciudadano. Por ello, el artículo 4 de la LOFAGE individualiza, resalta y especifica el principio de servicio a los ciudadanos.

Evidentemente, el destinatario de los servicios públicos, el ciudadano, es un agente menos homogéneo que el cliente de una organización privada, ya que, dada la diversidad y heterogeneidad de aquellos, puede revestir múltiples papeles, desde beneficiario de prestaciones a comprador, contribuyente o administrado, sometido a regulaciones, autorizaciones e inspecciones (SHAND y ARNBERG, 1996). Pero tales diferencias, por patentes que sean, no deben hacer diferente la actitud del prestador del servicio en unos casos y otros. En todos ellos, este ciudadano multifacético constituye un auténtico cliente, no porque pague una contraprestación -que no siempre lo hace de forma directa e inmediata-, sino porque es la razón de ser del servicio público.

\section{Misión de la Secretaría de Estado para la Administración Pública. El papel de la Inspección General de Servicios de la Administración Pública}

La Secretaría de Estado para la Administración Pública (SEAP), como órgano superior del Ministerio de Administraciones Públicas encargado de la dirección, impulso y gestión de las atribuciones relativas, entre otras, a la organización y racionalización de la Administración General del Estado, se sitúa obviamente en el plano de la macro-calidad (POLLT y BOtCKaEfT, 
1995). De acuerdo con esa posición competencial, la Misión de la SEAP, consiste en:

Impulsar y dirigir la organización y racionalización de. la Administración del Estado, a través de la optimización del uso de sus recursos y la mejora de las técnicas de gestión pública, en aras a lograr una Administración eficiente al servicio del ciudadano y adaptada al Estado autonómico y a la Unión Europea.

Así, las actuaciones de la Secretaría de Estado para la Administración Pública en materia de calidad no apuntan a la implantación de planes o programas de gestión de calidad en una/s organización/es propia/s prestadora/s de servicios directos a los ciudadanos, sino al desarrollo de la política global de calidad en los servicios públicos estatales como una estrategia para lograr los objetivos de Potenciar la transparencia y receptividad de la Administración General del Estado (AGE) bacia los ciudadanos y de Optimizar la utilización de los recursos bumanos y materiales de la $A G E$.

En este marco, la SEAP ejerce sus competencias en materia de calidad fundamentalmente a través de la Inspección General de Servicios de la Administración Pública (IGSAP). Este órgano directivo desarrolla al respecto actuaciones directas y acciones específicas de apoyo y colaboración técnica y formula programas y propuestas tendentes a la mejora de los sistemas de gestión pública y, por otra parte, impulsa y coordina las iniciativas de desarrollo del principio de servicio a los ciudadanos, mediante la elaboración y propuesta de programas de evaluación de la calidad de los servicios.

En definitiva, el planteamiento de la SEAP sobre la Calidad responde a un doble objetivo de mejorar los servicios prestados a los ciudadanos y de remover las ineficiencias burocráticas de cara a los agentes económico-sociales, confluyendo ambas vertientes en la meta última del bienestar y la competitividad internacional.

\section{Estrategia y actuaciones de la Inspección General de Servicios de la Administración Pública}

La Calidad no constituye una preocupación específica del sector privado ni del público. Mejor dicho, no existe una preocupación específica por la calidad en el mundo empresarial y otra preocupación específica en la Administración. La preocupa- ción es, en el fondo, la misma porque el contexto y los desafíos son comunes. Obviamente, hay diferencias entre un ámbito y otro (principios, valores, objetivos, etc.) pero, desde un punto de vista macro o nacional, las ráces y la finalidad úitima son las mismas. Como hemos visto: competitividad y bienestar.

Por ello, la IGSAP desarrolla su actividad de forma abierta y participativa, colaborando con un elevado número de Ministerios y Organismos públicos, así como con entidades asociativas privadas y organismos internacionales.

Desde 1993, la IGSAP participa activamente en la Asociación Española para la Calidad (AEC), en cuyo seno y a efectos de institucionalizar tal participación se creó una Sección de Calidad en las Administraciones Públicas (SECAP). Las actividades de este órgano, presidido y coordinado por la IGSAP y en el que se integran varias docenas de entidades públicas -estatales, autonómicas y locales - fueron presentadas en una Jornada celebrada en el INAP en 1995. Como frutos tangibles deben destacarse los siguientes:

* La Guia para la consideración de criterios de calidad en las adquisiciones públicas de suministros. Este documento, que pretende servir de orientación para tener en cuenta factores de calidad a la hora de tratar con los proveedores de la Administración, fue sometido en marzo de 1997 al escrutinio de un grupo representativo de responsables de la áreas de aprovisionamiento y servicios de distintas administraciones públicas, que lo acogieron favorablemente con el ánimo de aplicarlo en sus respectivas organizaciones.

* La Guía para la gestión de calidad en los servicios públicos. Adaptación de la norma ISO-9004.2. Este trabajo pretende facilitar la eventual aplicación de un modelo "normativo" de gestión de calidad en aquellos organismos que así lo estimen, sin que ello suponga decantarse por el mismo frente a otros modelos (EFQM, etc.), como se verá más adelante.

* Diseño de un Curso de Gestión de Calidad en los Servicios. Con un programa elaborado por la Sección e impartido por profesores seleccionados por la misma, de febrero a junio de 1997 se desarrolló en el INAP un acción formativa teórico-práctica de 140 horas, para normalizar y poner en común los conocimientos de una serie de funcionarios ya familiarizados con la gestión de calidad, de cara a convertirse en impulsores de su difusión en la Administración Pública.

* Partiendo de la experiencia anterior, la Sección ha diseñado un Curso de gestión de la calidad en la Administración Pública de 50 horas lectivas, destinado a directivos y cuadros, que desde 1999 se impartirá regularmente dentro de 
los programas anuales del INAP y, en su caso, de los centros de formación de las Comunidades Autónomas.

Desde 1994 ininterrumpidamente, la IGSAP también viene estando presente en el Congreso de Empresas de Calidad que anualmente organiza en Barcelona el Fơrum Qualitat, coordinando los talleres sobre Administración Pública. Además, en todas sus ediciones, ya sea directamente por funcionarios de la IGSAP, ya sea a través de otros miembros de la Sección de Administración de la AEC, se han presentado ponencias y comunicaciones, posteriormente publicadas.

Más recientemente, en marzo de 1997, junto con la Subsecretaría del Ministerio de Administraciones Públicas, la IGSAP se ha incorporado a otra importantísima institución promotora de la calidad. En el Club de Gestión de Calidad también se ha formado un foro de sector público que, además del Ministerio de Administraciones Públicas, cuenta con la presencia de destacadas organizaciones públicas.

Por lo que respecta al ámbito internacional, debe subrayarse la participación regular en el Comité de Gestión Pública (PUMA) de la OCDE, formando parte de la Service Quality Network y de la Performance Management Network. La vinculación activa a este marco de referencia, a este bencbmarking internacional, resulta prioritaria para orientar la labor de la IGSAP porque a(...) en toda Administración receptiva la mejora de la calidad del servicio es un aspecto fundamental de la gestión del rendimienton.

La IGSAP, por otra parte, articula sus actuaciones en dos grandes líneas estratégicas que convergen hacia los objetivos antes mencionados: Optimizar la utilización de los recursos bumanos y materiales de la AGE y Potenciar la transparencia y receptividad de la AGE hacia los ciudadanos. La primera de las líneas estratégicas comprende, por una parte, programas o proyectos de Mejora de los sistemas de gestión pública y, por otra, de Impulso y evaluación de la calidad de los Servicios Públicos. La segunda línea estratégica incluye dos bloques de actuaciones tendentes al Acercamiento al ciudadano y a la Simplificación y racionalización.

\subsection{Programas de optimización de recursos humanos y materiales de la AGE}

\subsubsection{Programas en materia de mejora de los sistemas de gestión pública}

A) La IGSAP desarrolla actuaciones de consultoría directa en numerosas organizaciones públicas para promover procesos de cambio, facilitando altermativas de mejora en su funcionamiento. Entre los proyectos ejecutados, cabe mencionar los trabajos en el Consejo General del Poder
Judicial, en el INAEM, en la Dirección General de Tráfico, en la UNED, en la Universidad de Cádiz, etc.

\section{B) Evaluación del rendimiento}

Nuestra Administración está obligada a adecuar su actuación a los principios de eficacia, eficiencia y economía, y como consecuencia de la entrada en vigor de la nueva Ley de Organización y Funcionamiento de la Administración General del Estado debe ser dirigida con los principios de programación por objetivos y control de los resultados y costes derivados de la gestión. Este mandato legal se ve reforzado, por otra parte, en el momento actual, por la necesidad de contribuir a los objetivos derivados del Plan de Convergencia, racionalizando al máximo la utilización de los recursos económicos puestos a disposición de las Administraciones Públicas. Para asegurar el cumplimiento de este doble objetivo, se pondrá en marcha en la Administración General del Estado un Programa de Evaluación del Rendimiento de los Organismos y Servicios Integrados en la Administración General del Estado, que introduzca en los sistemas de dirección pública la evaluación de los resultados, a través de un cambio en las prácticas administrativas y gestoras.

De acuerdo con el programa elaborado por el Gobierno, todos los órganos de la Administración General del Estado deberán incorporar a la gestión ordinarià el sistema de evaluación del rendimiento. Este sistema está concebido como un instrumento flexible de control y evaluación de la gestión que permitirá a los directivos y gestores de la Administración General del Estado medir la eficacia, la eficiencia, los costes y la calidad de su gestión, a través de la evaluación sistemática y continua de la relación existente entre los resultados obtenidos y los medios precisos para su consecución. Todo ello con la finalidad de generar una cultura orientada al resultado, conseguir una mayor eficiencia en el uso de los recursos y mejorar la calidad de los servicios que se prestan a los ciudadanos.

\subsubsection{Programas de impulso y evaluación de la calidad de los servicios públicos}

A) Realización de diagnósticos de calidad, con el MODELO SERVQUAL, en el Decanato de los Juzgados de Valencia y en el Centro de Información Administrativa (CIA), dependiente de la propia Dirección General de la IGSAP. Los trabajos concluyeron en el primer caso con la presentación de un informe de evaluación sobre calidad percibida que, entre otras cosas, puso de manifiesto la pertinencia y viabilidad de los métodos de gestión de calidad incluso en órganos tan apeculiares。 como los judiciales. 
B) El proyecto denominado SERVICIO A CIUDADANOS tiene como objetivo elaborar una norma reguladora de los sistemas de determinación de estándares de calidad, evaluación y mejora de los servicios de la ACE, así como una guía para su implantación. Como puede adivinarse, se trata de una iniciativa de envergadura porque, aunque parte del Ministerio de Administraciones Públicas desde su responsabilidad horizontal, afecta a la generalidad de la Administración General del Estado, requiere su consenso y conlleva un proceso dilatado y complejo hasta su promulgación.

C) El proyecto bautizado Modelo EUROPEO tiene como objetivo la elaboración de una guía y los materiales para utilizar el Modelo Europeo sobre autoevaluación en las unidades administrativas.

Como todos saben, la gestión de calidad constituye una nueva orientación del funcionamiento de las organizaciones que está permitiendo su progreso en los últimos años. La creación de la Fundación Europea de la Gestión de Calidad ha hecho posible que se impulse de forma decidida el avance de la mejora de las organizaciones, puesto que el Modelo Europeo de gestión de calidad, que ha elaborado la Fundación, ha integrado los componentes esenciales de la gestión de la calidad en un paradigma que permite su utilización y aprovechamiento por cualquier tipo de empresa u organización.

El Modelo Europeo tiene un carácter global que alcanza todos los aspectos del funcionamiento de una organización. Permite, por lo tanto, efectuar una aproximación integral a todos sus procesos de desarrollo y a todos los niveles de su estructura. Todo ello dentro de un marco de referencia que se basa en los conceptos fundamentales de la gestión de calidad. De tal forma que el análisiş y la transformación de las organizaciones realizadas desde esta perspectiva se apoyan en unos principios que dotan de sentido las acciones que se lleven a cabo.

\subsection{Programas para potenciar la transparencia y receptividad de la Administración General del Estado hacia los ciudadanos}

\subsubsection{Programas de acercamiento al ciudadano}

Como ya se ha señalado repetidamente, la concepción de la simplificación administrativa no se agota en la enunciación de grandes principios, y tampoco siquiera en la adopción de medidas legales o reglamentarias cuya aplicación puede dejar mucho que desear. Gran parte de las medidas de simplificación se plasman en actuaciones concretas con resultados medibles y evaluables.
Entre tales proyectos, parece de interés el dedicar una relevante atención a una serie de ellos que a continuación se exponen:

A) El programa «ventanilla única.

El programa tiene como factores más relevantes el papel protagonista a desempeñar por las Entidades Locales, en su condición de Administración más cercana a amplios sectores de la ciudadanía; la finalidad de acercamiento de la Administración a los ciudadanos y la previsión de una utilización intensiva de las tecnologias de la información y la comunicación, sin cuya existencia el proyecto hubiera sido, sino inviable, enormemente dificultoso y con elevados costes en infraestructura y medios.

Pero además, el programa ventanilla única tiene como nota definitoria la de constituir una actuación basada en la cooperación entre los tres niveles administrativos que coexisten en nuestro pais: La Administración General del Estado (AGE), las Comunidades Autónomas y las Entidades Locales.

Aun cuando su desarrollo ha trascendido en mucho a las causas que dieron lugar a su nacimiento, es preciso señalar que el programa "ventanilla única" nace originariamente para dar respuesta a la demanda (manifestada a través del Defensor del Pueblo y de numerosas reclamaciones) de los habitantes de pequeños municipios que encuentran dificultades para relacionarse con la AGE y con la Administración autonómica debido a la inexistencia en ellos de dependencias de dichas Administraciones. Tal circunstancia implica para los vecinos de esos municipios la necesidad de afrontar gastos, desplazamientos, etc., para realizar cualquier gestión; o bien les obliga a acudir a intermediarios profesionales en su relación con las grandes Administraciones territoriales.

El Gobierno potenció enormemente los instrumentos previstos en el Acuerdo del Consejo de Ministros de 23 de febrero de 1996; y a estos efectos ha de reseñarse que se suscribieron -a fecha 29 de abril de 1997- un total de 265 Convenios bilaterales con Entidades Locales.

Pero, simultáneamente, el Gobierno tomó conciencia de que el programa requería un nuevo enfoque que subsanase las carencias de su definición inicial. Para ello, adoptó el Acuerdo del Consejo de Ministros de 4 de abril de 1997, que amplía los objetivos del propio programa en una triple perspectiva (posibilitando la participación con carácter simultáneo en el proyecto de las Comunidades Autónomas; procurando la normalización del entero proceso, mediante la fijación de criterios uniformes; y asentando las bases de lo que en el futuro constituirá no sólo un sistema registral intercomunicado sino también un sistema registral informatizado). 
Buena muestra del éxito de la segunda fase del programa - los Convenios Marco- es la participación, a menos de un año de su inicio, de 9 CC.AA. (Castilla y León, Cataluña, Valencia, Madrid, Galicia, Extremadura, Canarias, Castilla-La Mancha y Baleares) que pronto se verán incrementadas con Murcia. No obstante, el Gobierno confía en que paulatinamente se produzca la incorporación de todas las CC.AA. a un programa basado en la cooperación entre los tres niveles administrativos -estatal, autonómico y local一.

Pero, sobre todo, el dato más alentador es el de la participación de cerca de 800 Entidades Locales, lo que supone un ritmo vertiginoso y un buen indicador del éxito del programa que responde a una pluralidad de objetivos.

Tales objetivos son los siguientes:

* La babilitación de los registros de las Entidades Locales para recibir comunicaciones dirigidas a la AGE y a la correspondiente Comunidad Autónoma.

* El segundo objetivo es el de lograr la interconexión informática de los registros, de forma que en un futuro no muy lejano la entrada de una comunicación en cualquier registro de las Administraciones participantes en el proyecto sea conocida en tiempo real en el punto de destino de dicha comunicación.

* Un tercer objetivo pretende que en cualquier punto del mismo pueda tenerse acceso a la información general de cualquier Administración participante.

* El último objetivo atiende a un factor de complejidad que es consecuencia de un Estado con distintas Administraciones Públicas: la existencia de procedimientos en los que la tramitación se reparte entre las distintas Administraciones, e incluso de grupos de procedimientos con objetos comunes que, igualmente, son responsabilidad de diferentes Administraciones.

\section{B) La acción PISTA}

Pero lo que se pretende es que el programa ventanilla única no se quede en un mero "proyecto" como tantos otros, cargado de buenas intenciones que nunca llegan a ejecutarse y son progresivamente olvidadas. Como se ha reiterado, el programa tiene como componente fundamental una intensiva utilización de las tecnologias de la información y la comunicación.

La Administración General del Estado pone a punto ya instrumentos en que comienzan a plasmarse los objetivos señalados. Así, desde el pasado diciembre cualquier ciudadano puede obtener en Internet información acerca de cualquiera de los procedimientos que la AGE tramita (http:t/www.igsap-map.es).
Proximamente, podrá también imprimir su propia solicitud y -en el futuro- incluso podrá remitirla por correo electrónico desde su propio domicilio o desde cualquiera de los puntos de la red que se esta constituyendo.

La acción PISTA impulsada y financiada por el Ministerio de Fomento y desarrollada por el Ministerio de Administraciones Púiblicas se despliega en dos subproyectos; orientándose el primero de ellos a lograr la interconexión de los registros (transmisión de asientos y documentos) y estando el segundo destinado a proporcionar herramientas tecnológicas que permitan la difusión integrada de información, la transmisión de modelos de solicitudy la teletramitación de determinados procedimientos. En 15 meses se desarrollarán experiencias piloto en los ámbitos señalados, y las Administraciones participantes dispondrán gratuitamente de todos estos productos. El ciudadano verá como el programa "ventanilla única paulatinamente se convierte en una realidad que simplifica y mejora sus relaciones con las Administraciones Públicas.

\section{C) El proyecto CERES (Certificación Española)}

Como ha podido comprobarse a lo largo de la exposición, el programa "ventanilla única" tiene como uno de sus ejes centrales el de la utilización intensiva de la tecnología de la información y la comunicación; eje por lo demás común a cualquier medida de simplificación que haya de adoptarse en el umbral del Siglo XXI. La acción PISTA constituye la demostración más evidente de esa afirmación.

Sin embargo, la utilización de tales tecnologías en el proyecto "ventanilla única" no puede sustraerse a lo que unánimamente es considerado como el mayor de los problemas que afecta a aquellas. Nos referimos al problema de la seguridad.

La cuestión se concreta en asegurar que las comunicaciones por medios telemáticos en las que se concreta el programa "ventanilla única" dispongan de un nivel de seguridad que garantice el cumplimiento de los requisitos normativos para que dichas comunicaciones sean válidas y eficaces. Tales requisitos, contemplados en el artículo 45 de la LRJ-PAC, pueden sintetizarse en la integridad, confidencialidad y autenticidad de unas comunicaciones cuyo contenido tiene importantes repercusiones jurídicas tanto para el ciudadano como para la Administración.

Debido a lo expuesto, el programa "ventanilla única" tiene como uno de sus referentes otro proyecto cuyo objeto es el de garantizar la seguridad de las comunicaciones en los términos expresados. El proyecto es el denominado CERES (Certificación española). 
El proyecto CERES, en el que participan la Fábrica Nacional de Moneda y Timbre, el Ministerio de Economía y Hacienda, el Ministerio de Administraciones Públicas y el organismo Correos y Telégrafos, se apoya en el impulso a la utilización de las tecnologías de la comunicación que representan normas como el artículo 45 de la LRJ-PAC y el Real Decreto 623/1996. Dichas normas han terminado con la disociación entre normativa y realidad que se deducía de unas administraciones con un elevado nivel de equipamiento informático que - por imperativos jurídicos - no podía ser plenamente utilizado.

\subsubsection{Programas de simplificación y racionalización}

Podemos definir la racionalización como el conjunto de medidas de mejora de los procesos de gestión en la actividad administrativa con los siguientes objetivos:

* La reducción de los tiempos de respuesta al usuario, que repercute en disminución de los costes para el sector privado y en una agilización de la satisfacción de sus demandas.

* La transparencia en la gestión de los procedimientos, articulando instrumentos concretos de participación e información del ciudadano y para la aplicación efectiva de sus derechos.

* La reducción de trámites, eliminando todas aquellas actividades que consumen recursos sin aportar valor añadido a los procesos.

* La incorporación de las tecnologías de la información y la comunicación a la gestión, incidiendo en la tramitación automatizada de los procedimientos y en la aplicación de técnicas y medios informáticos, electrónicos y telemáticos a las relaciones con los ciudadanos.

Como resulta evidente, el principal objeto de la racionalización es el procedimiento administrativo, en su acepción como proceso de toma de decisiones en la organización administrativa.

La racionalización persigue por tanto reducir los efectos disfuncionales para, por una parte, conseguir una mejora significativa de la calidad del servicio prestado a los ciudadanos y, por otra, lograr el mantenimiento e incluso la disminución de los costes en recursos bumanos y medios materiales como ayuda para la necesaria restricción del gasto público. Ambas finalidades son complementarias, sin que la consecución de una de ellas pueda restringir o menoscabar a la otra.

\section{DiAGNÓSTICO}

Del examen y análisis de los datos sobre procedimientos actualmente vigentes en la Administración General del Estado cabe extraer unas conclusiones que ofrecen, en síntesis, un diagnóstico de la situación actual de la actividad administrativa para delimitar el ámbito de operatividad de los instrumentos de desregulación y simplificación que la propia Ley de Régimen Jurídico de las Administraciones Públicas y del Procedimiento Administrativo Común (LRJ-PAC) y —sobre todo- su reforma en curso presentan.

La primera conclusión que se extrae respecto del número de procedimientos es la sospecha de la existencia de una excesiva formalización, reglamentación e intervención administrativa en las actividades privadas. A ello apunta no sólo el a todas luces elevado número de procedimientos (2.080), sino el que dentro de ellos la categoría predominante sea aquella cuyo objeto es el condicionamiento y sujeción a requisitos de las actividades de ciudadanos y empresas - (Autorizaciones 33\%)-.

Si bien ha de tenerse la suficiente prudencia para no calificar de innecesarias tantas autorizaciones (la gran mayoría de las cuales sin duda responde a necesidades y justificaciones muy razonables), lo que resulta evidente es que se impone una revisión de las causas de la presencia de una variedad tan enorme de procedimientos autorizatorios diferenciados, muchos de ellos con sus respectivas normas de referencia.

En su conjunto aparecen como un intrincado entramado burocrático frente al ciudadano y en general frente a quienes - como las PYME - dependen de la accesibilidad de dichos procedimientos para la iniciación y desarrollo de sus actividades. Las consecuencias en cuanto a costes públicos - el mantenimiento de una infraestructura administrativa especializada en el conocimiento y gestión de las numerosas especialidades - y privados - los derivados de los esfuerzos para superar y acceder a las decisiones administrativas-, aun siendo hoy imposibles de determinar se adivinan cuantiosas.

Una segunda consecuencia resulta de la terminante mayoría de procedimientos que tienen atribuidos efectos presuntos desestimatorios (silencio negativo 76,4\%). Ese dato estadístico es revelador del fracaso en este punto de una Ley cuya declarada vocación era la de la instauración del silencio positivo, sin duda debido a la habilitación a las normas reglamentarias para establecer supuestos de desestimación.

El silencio positivo, o la estimación presunta en terminología de la propia Ley 30/1992, es un elemento clave en una visión liberalizadora de la actividad administrativa ya que supone la concesión de prioridad a la iniciativa ciudadana cuando se produce la inactividad de la Administración (piénsese en la no resolución de una solicitud de licencia de la que puede depender el inicio de una actividad y la generación del empleo correspondiente.

Finalmente, una tercera conclusión se refiere al tema de los plazos de respuesta al ciudadano. Si bien los datos no son del 
todo desalentadores - un 60\% de los procedimientos se resuelve en plazos inferiores a los tres meses-, lo cierto es que cualquier esfuerzo en este sentido ha de ser adecuadamente impulsado. En una etapa en la que la información, la comunicación y las decisiones y transacciones inherentes a cualquier actividad se desenvuelven a velocidades cada vez mayores; la propia sociedad demanda respuestas inmediatas del aparato administrativo. Parafraseando el tantas veces repetido dicho acerca de la justicia, una Administración lenta no es una verdadera Administración.

\section{RACIONALIZACIÓN Y REFORMA DE LA LEY 30/1992}

\section{CREACIÓN DE Una COMISIÓN DE RaCiONaLIZACiÓN}

Un primer instrumento de racionalización previsto en la aludida reforma es la Comisión Interministerial que establece la disposición adicional única del proyecto de Ley de reforma, a la que se atribuye una misión concretada en la consecución de diversos objetivos de desregulación y simplificación que atienden a los aspectos centrales del diagnóstico que se acaba de establecer.

Del apartado primero de la disposición resulta con claridad un nítido objetivo de racionalización normativa, en el sentido de reforma y gestión de la reglamentación que constituye la acepción consolidada de dicho concepto.

Dicho objetivo es el de la implantación de categorias generales de procedimientos; que responde al hecho de la existencia de un muy elevado número de procedimientos (2.080) y a las consecuencias disfuncionales que se derivan de ese dato (dispersión de actividades administrativas, excesiva reglamentación específica, incidencia en costes públicos y privados) que ya fueron comentados al exponer el diagnóstico de la actual situación.

El mencionado objetivo lleva aparejado -como inevitableotro no menos importante que es el de la reducción y sistematización de las normas reguladoras de tales procedimientos; expresado con ese doble contenido porque si bien es complicado alcanzar grandes resultados de reducción normativa (habida cuenta de que en nuestro sistema jurídico las regulaciones procedimentales suelen encontrarse insertadas en las correspondientes regulaciones sustantivas, que son difícilmente afectables), lo que sí parece posible es lograr una sistematización de la dispersa normativa procedimental facilitando su accesibilidad y claridad para el ciudadano.

Pero la Comisión ostenta también funciones de racionalización de la gestión, entre las que cabe destacar dos cuya incidencia en los procedimientos administrativos -por tanto, en las relaciones del ciudadano con la Administración- determinará una mayor agilidad en los tiempos de respuesta y una flexibilización en la iniciación y desarrollo de actividades privadas.

Así, se menciona expresamente la reducción de trámites innecesarios. Como toda organización burocrática la Administración Pública tiende a la retroalimentación, es decir, a la generación de actividades que justifiquen su existencia y aumenten su dimensión. Como consecuencia, en los procedimientos habitualmente se contienen trámites innecesarios - pudiéndose definir como aquellos que no responden a finalidades inherentes al objeto procedimental, y por tanto no aportan valor añadido alguno a la decisión final-.

La Comisión deberá detectar tales trámites y proponer su anulación. Como consecuencia de tal anulación, aunque no explicitada en la disposición, se debe producir una reducción del tiempo de respuesta de la Administración. Es decir, del tiempo de resolución de los procedimientos que resulten afectados por la consecución de este objetivo.

Pero quizás el elemento central en las funciones de la Comisión con consecuencias simplificadoras sea la expansión del silencio positivo, contemplado como principio de la reforma con las adecuadas garantías - reserva de ley para establecer el supuesto contrario-.

El silencio positivo, como ya se señaló, es un elemento clave en una visión liberalizadora de la actividad administrativa ya que supone la concesión de prioridad a la iniciativa ciudadana cuando se produce la inactividad de la Administración. Su expansión, unida a la reducción de los tiempos de respuesta, flexibilizará y dinamizará enormemente las relaciones ciudadano-Administración, con efectos trascendentales en el crecimiento económico y en los costes marginales de los agentes económicos. En este aspecto, cabe esperar que los trabajos de la Comisión consigan resultados significativos y no se vean frustrados por la habitual resistencia burocrática de los órganos administrativos.

\footnotetext{
Director General de la Inspección General de Servicios de la Administración Pública (MAP).
} 


\section{Bibliografia}

AEC/SECAP (1997), Guia para la consideración de criterios de calidad en las adquisiciones puiblicas de suministros (Mimeo). AEC, Madrid.

-(1997), Guía para la gestión de calidad en los servicios puíblicos. Adaptaciōn de la norma ISO-9004.2 (Mimeo). AEC, Madrid.

__(1995), ·Presentación de la Sección de Calidad en las Administraciones Públicas. Gestión y evaluación de la calidad en los senvicios priblicos. MAP, Madrid.

BouckaERT, Geert (1996), •Commentaires sur l'étude (...) Informer les clientes (...). L'Administration à l'écoute du public. OCDE, Paris.

DuPuIs, Jérôme (1991), Le contrôle de gestion dans les organisations publiques. PUF, Paris.
LABOVITz, Chang y Rosaisky (1995), Cómo bacer realidad la calidad. Díaz de Santos, Madrid.

LŌFFER, Elke (1996), ‘la modemización del sector público desde una perspectiva comparativa.... Documentos INAP, n.. 8, INAP, Madrid.

OCDE (1988), La Administración al servicio del Püblico. MAP, Madrid

POLutT, C. y BoucKaERT, G, de (1995), Quality Improvement in European Public Services. Sage, Londres.

SHAND, D. y ARNBERG, M. (1996), ‘Document de référence. L' Administratión à l'écoute du public. OCDE, Paris. 


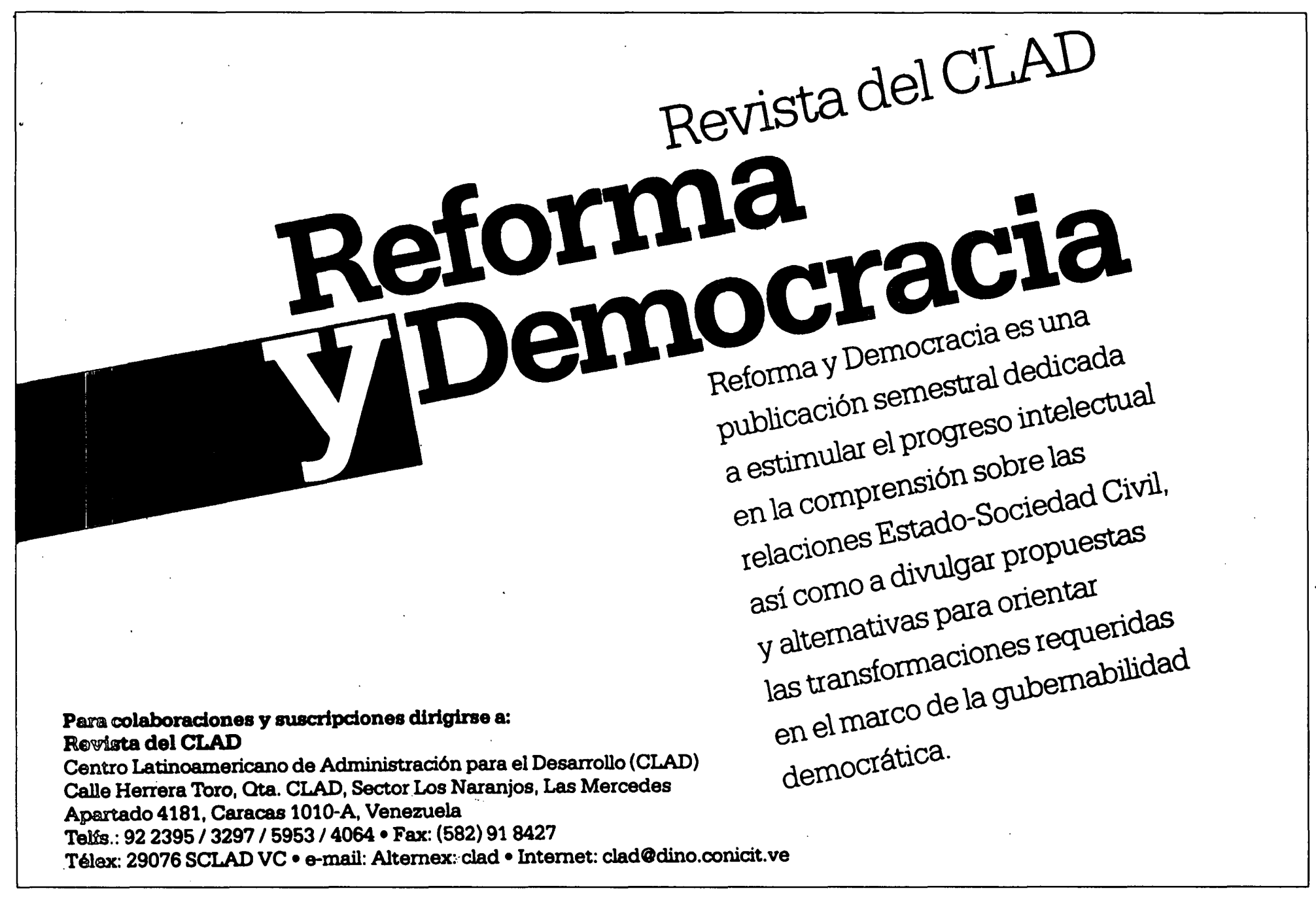

